\title{
14 Die absolute Reflexion als Ergebnis und Anfang
}

Hegel führt in den dritten Abschnitt des ersten Kapitels der Wesenslogik ein, indem er „das Wort der fremden Sprache“ (GW 11, S. 249), den Terminus „Reflexion“ also, der bereits das letzte Wort des zweiten Abschnitts gewesen ist, auf äußerst abstrakte Weise und in denkbar komprimierter Form erörtert, bevor er sich dann in drei Unterabschnitten den einzelnen Stufen der Reflexionsformen zuwendet. Adorno zitiert am Anfang seines Hegel-Essays Skoteinos oder Wie zu lesen sei ein charakteristisches Satzgefüge eben dieser kurzen Einführungsabschnitte, um daran zu verdeutlichen: „Im Bereich großer Philosophie ist Hegel wohl der einzige, bei dem man buchstäblich zuweilen nicht weiß und nicht bündig entscheiden kann, wovon überhaupt geredet wird, und bei dem selbst die Möglichkeit solcher Entscheidung nicht verbrieft ist. " ${ }^{100}$ Wenn eben diese Sätze, die die in der Forschung vieldiskutierte Wendung „Das Werden im Wesen, seine reflectirende Bewegung, ist daher die Bewegung von Nichts $\mathrm{zu}$ Nichts, und dadurch zu sich selbst zurück“ (GW 11, S. 250) enthalten, in isolierter Form tatsächlich eine Art von Unzugänglichkeit nachzuweisen scheinen, so klärt sich der gesamte Abschnitt im Zusammenhang einer genauen Interpretation, wie sie auch hier versucht wird, jenseits aller angeblichen Dunkelheit doch auf. Hegel kommt es darauf an, die ganz eigensinnige Art und Weise, wie er den stark vorbesetzen, durch traditionelle Bedeutungen vereinnahmten Begriff der Reflexion analog zu seiner Neuinterpretation des Scheins - umzubesetzen gedenkt, deutlich zu machen: Überhaupt ist das „Erste Kapitel“ der Wesenslogik davon geprägt, dass tragende Termini der abendländischen Metaphysik wie Wesen, Schein und Reflexion von Hegel gegen den Strich gelesen werden, um sie einer erweiterten Funktionalität zugänglich zu machen, die imstande wäre, die ,alten' Probleme der abendländischen Ontologie zu lösen.

Hegel weicht also von den gewöhnlichen Vorgaben hinsichtlich dessen, was „Reflexion“ philosophisch meint, deutlich ab. Mindestens zwei Transformationsrichtungen sind dabei zu unterscheiden, die allerdings beide darin übereinkommen, den Begriff jenseits seines konventionellen Themenfeldes von Subjektivität und Bewusstsein neu zu verorten. „Es ist aber hier nicht, weder von der Reflexion des Bewußtseyns, noch von der bestimmtern Reflexion des Verstandes, die das Besondere und Allgemeine zu ihren Bestimmungen hat, sondern von der

100 Theodor W. Adorno: Zur Metakritik der Erkenntnistheorie. Drei Studien zu Hegel, Frankfurt am Main 2003, S. 326.

Ә OpenAccess. (c) 2021 Claudia Wirsing, publiziert von De Gruyter. (cc) BY unter einer Creative Commons Namensnennung 4.0 International Lizenz. 
Reflexion überhaupt die Rede.“ (GW 11, S. 254) Reflexion meint hier also weder allgemeiner die Denktätigkeit des Verallgemeinerns überhaupt (im Sinne von „über etwas reflektieren“), ${ }^{101}$ noch spezifischer die Denktätigkeit des Selbstbewusstseins, sich seiner eigenen Denkhandlungen bewusst zu sein (im Sinne von „reflexiv denken“). ${ }^{102}$ Mit Reflexion sind also hier im Rahmen der Wesenslogik keine „epistemische[n] Tätigkeiten eines Subjekts im Sinne inneren Handelns“103 geimeint, sondern wie Michael Quante zu Recht feststellt, „eine das Subjekt allererst konstituierende Reflexion, die nicht selbst schon nach dem Modell absichtlichen Handelns konzipiert werden darf.“104 „Reflexion überhaupt“ als die „reine absolute Reflexion“ (GW 11, S. 250), ganz isoliert als Ergebnis der bisherigen Überlegungen genommen, bezeichnet hier vielmehr die bis zu diesem Punkt herausgearbeitete Logik von Bestimmtheit überhaupt im Wesen. ${ }^{105}$ Hegel macht dies ganz klar, indem er der Art von Bestimmtheit im Wesen, welche die Reflexion ist, die Art der „Bestimmtheit überhaupt“ aus dem Sein nochmals kurz und prägnant gegenüberstellt: „In dem Werden des Seyns liegt der Bestimmtheit das Seyn zu Grunde, und sie ist Beziehung auf Anderes.“ (GW 11, S. 239) Dem „Werden“ des Seins steht die „Bewegung“ der Reflexion gegenüber: Beide kommen darin überein, „Bestimmtheit überhaupt“ als je verschiedene Weisen von Prozessualität zu verstehen. „Bestimmtheit überhaupt“ ist Bestimmtwerden bzw. sich vollziehendes Bestimmen. Im Wesen ist die Reflexion die Form, wie das Wesen a) strukturell in sich selbst mit sich in seinem Anderssein zusammenhängt,

101 „Die Reflexion wird gewöhnlicher Weise in subjectivem Sinne genommen, als die Bewegung der Urtheilskraft, die über eine gegebene unmittelbare Vorstellung hinausgeht, und allgemeine Bestimmungen für dieselbe sucht oder damit vergleicht.“ (GW 11, S. 254, Anm.)

102 Vgl. zu den Bedeutungen von „Reflexion“ Iber: Metaphysik absoluter Relationalität, S. 122f., $132 \mathrm{f}$.

103 Michael Quante: „Die Lehre vom Wesen. Erster Abschnitt. Das Wesen als Reflexion in ihm selbst.“, in: Kommentar zu Hegels Wissenschaft der Logik, hg.v. Michael Quante und Nadine Mooren unter Mitarbeit v. Thomas Meyer und Tanja Uekötter, Hegel Studien, Beiheft 67, Hamburg 2018, S. 275-324, hier: S. 293, Fußnote 19.

104 Quante: Die Lehre vom Wesen, S. 293, Fußnote 19.

105 Ich folge hier Iber, der, gegen große Teile der älteren Forschung (vgl. Iber: Metaphysik absoluter Relationalität, S. 133f.), die dezidiert nicht-subjektive, rein logische Struktur der Reflexion bei Hegel herausarbeitet und weitergehend (für unsere Frage aber ohne Belang) diese subjektlose objektive Struktur der Reflexion als Lösungsmöglichkeit sowohl gegenüber den Aporien der Selbstbewusstseinstheorien des Idealismus, wo sie sich auf Reflexivität stützen müssen (Iber: Metaphysik absoluter Relationalität, S. 135-138), als auch gegenüber der ebenfalls subjektlosen Identitätsphilosophie Schellings ansieht (Iber: Metaphysik absoluter Relationalität, S. 139-141). Seine Lesart trifft sich darin mit meiner, die Form und Funktion von Reflexion im Wesen als ein den Gegensätzen von Subjekt und Objekt, Ich und Welt selbst in ihrer einfachsten reinen Form noch vorgelagertes generatives Geschehen zu begreifen. 
b) funktional dadurch mit sich vermittelt ist und c) begrifflich-semantisch in dieser Vermittlung der eigene Grund der Bestimmtheiten ist, die in ihm anzusiedeln sind. D.h. die Refelxion ist die wesenslogische Art und Weise, wie Unmittelbarkeit und Vermittlung, Positivität und Negativität, Selbstbezüglichkeit und Beziehung-auf-Anderes in der besonderen Form ihres inneren Zusammenhangs der „unendliche[n] Bewegung“106 (GW 11, S. 249) einer Selbstbeziehung im Anderssein eine neue Grammatik der Bestimmtheit (also der Bestimmtheit von „Bestimmtheit überhaupt“) ermöglichen. Unmittelbarkeit als das Andere zum Wesen, als reine negative Vermittlung, ist Schein, reflektierte Unmittelbarkeit, weil sie nichts anderes als das Wesen ist bzw. das Wesen als Anderes, das so in sich selbst scheint, d.h. in sich reflektiert ist, wo es sich auf die Unmittelbarkeit gegen sich bezieht. Dergestalt wird also die bereits ausführlich erläuterte Generativität von Unmittelbarkeit und sich negierender Negation auseinander als Ganzes des Wesens als „Reflexion“ bezeichnet, „als die unendliche Bewegung in sich, welche seine Unmittelbarkeit, als die Negativität, und seine Negativität als die Unmittelbarkeit bestimmt und so das Scheinen seiner in sich selbst ist" (GW 11, S. 249). Reflexion ist mithin

die Bewegung des Werdens und Uebergehens, das in sich selbst bleibt; worin das unterschiedene schlechthin nur als das an sich negative, als Schein bestimmt ist. [...] Die reflectirende Bewegung [...] ist das Andre als die N e g a ti on an sich, die nur als sich auf sich beziehende Negation ein Seyn hat. (GW 11, S. 249)

Christian Iber hat richtig festgestellt, dass Hegel hier seinslogische Termini („Übergehen“ - „Werden“ - „Anderes“) zur Beschreibung einer wesenslogischen Struktur benutzt. ${ }^{107}$ Seine Charakterisierung dieses Vorgehens als „inadäquat“ in Bezug auf den immanenten Stand der Begriffsbildung in der Logik sicherlich berechtigt -, verkennt allerdings, dass hier erneut mit großer Konsequenz die iterativ-regenerative Logik des Scheins in der Terminologie für das Wesen aufgenommen und damit die seinslogische Verantwortlichkeit des Denkens, wie es sich im Schein herausgebildet hat, ernst genommen wird. Wenn es als ein Ziel dieses Kapitels angesehen werden kann, nicht nur die rein prozessuale Form wesenslogischer Grammatik als den Begriff des Wesens selbst, sondern auch die minimale Form kategorialer „Realität überhaupt“ herauszuarbeiten, welche die Struktur des Verhältnisses letzter Bestimmungsrichtungen und ihrer irreduziblen

106 Unendlich ist diese Bewegung nicht im Sinne von endloser, sich in schlechter Unendlichkeit ohne Ergebnis vollziehender, sondern als eine solche, die zwischen Momenten verläuft, die in der Bewegung in ihr Anderes kein Anderes, sondern sich selbst erreichen.

107 Iber: Metaphysik absoluter Relationalität, S. 122. 
Abhängigkeit voneinander meint, dann wird eben die Beachtung dieser seinslogischen Verantwortlichkeit - und damit der normativen Funktion dieser Begriffsbildung - eine tragende Funktion haben müssen.

In einer längeren, erneut sehr dichten und komplizierten Passage dieses Einleitungsabschnitts in die Reflexionsformen gibt Hegel ferner noch einmal eine Art Überblick über die Arten und Funktionen der Negativität des Wesens:

\begin{abstract}
Die reflectirende Bewegung hingegen ist das Andre als die N e g a ti o n a n sich, die nur als sich auf sich beziehende Negation ein Seyn hat. Oder indem diese Beziehung auf sich eben diß Negiren der Negation ist, so ist die Negation als Negation vorhanden, als ein solches, das sein Seyn in seinem Negirtseyn hat, als Schein. Das Andere ist hier also nicht das Seyn mit der Negation oder Grenze, sondern die Negation mit der Negation. Das Erste aber gegen diß Andere, das Unmittelbare oder Seyn, ist nur diese Gleichheit selbst der Negation mit sich, die negirte Negation, die absolute Negativität. Diese Gleichheit mit sich oder Unmittelbarkeit ist daher nicht ein erstes, von dem angefangen wird, und das in seine Negation überginge, noch ist es ein seyendes Substrat, das sich durch die Reflexion hindurch bewegte; sondern die Unmittelbarkeit ist nur diese Bewegung selbst. (GW 11, S. 249f.)
\end{abstract}

Hegel unterscheidet jetzt zwei Aspekte am Fundament der Struktur des Wesens, nämlich an der „sich auf sich beziehenden Negation“, also an der reinen wesenslogischen Vermittlungsform reflexiver Selbstbezugnahme des Negativen, das sein Sein rein in diesem negierenden Selbstbezug hat. Damit fragt er nach dem Reflexiven, welches die Reflexion als Verhältnis sich negierender Negation ausbildet: Er bestimmt also, was das Reflexive an der Struktur der Reflexion ist. Bezugnehmend auf eine frühere Erörterung der vorliegenden Arbeit könnte man außerdem sagen, dass Hegel dabei näherhin die Aspekte von (1.) Selbstbezüglichkeit und (2.) Selbstreferenzialität der sich negierenden Negation unterscheidet: dass er also die Arten von Reflexivität an der Reflexion durchdenkt.

(1.) Unter der Hinsicht der Selbstbezüglichkeit der reflexiven Negativität betont Hegel ihren „Gleichheitsaspekt“ bzw. den Aspekt der Unmittelbarkeit an ihr: Als „negierte Negation“108 (oder, etwas unglücklich hier, weil eigentlich für die Einheit beider reserviert, als „absolute Negativität“) ist die Unmittelbarkeit im Wesen, die ja eigentlich gegen das Geschehen negierender Vermittlung steht, nichts anderes als das Mit-sich-gleich-Sein der Negation im Vollzug ihres negierenden Selbstbezuges. Selbstbezüglich ist die Reflexivität der Negation demnach, wo die „Bewegung selbst“ der sich negierenden Negation eine Unmittelbarkeit als Sichselbst-Gleichheit bedeutet, weil diese die Beschreibung ihres Selbstverhältnisses

108 „Negierte Negation“ deshalb, weil die Funktion des Negierens als Anderswerdens hier zugunsten des Gleichheitsaspekts der Negation mit sich hintangestellt wird. 
ausmacht: Unmittelbarkeit geht demnach der reflexiven Negativität nicht voraus oder in sie als Anderes hinein (,nicht ein e r s te s, von dem angefangen wird, und das in seine Negation überginge, noch ist es ein seyendes Substrat, das sich durch die Reflexion hindurch bewegte“, GW 11, S. 249f.), sondern ist wesenslogisch nur als Selbstbeschreibung der reflexiven Negativität zu fassen.

(2.) Unter der Hinsicht der Selbstreferenzialität hingegen, also unter dem Aspekt der reflexiven Selbstanwendung der Funktionen der Negation auf sich, ergibt sich für Hegel der Ungleichheitsaspekt reflexiver Negativität: Das „Negieren der Negation“, in welchem sich die Negation scheinbar in ihr Gegenteil aufhebt, als „Negation als Negation“ bzw. „Negation mit der Negation“ bezeichnet, und in welchem sie so in ihrem gesetzten Anderen ungleich mit sich zu werden scheint, erläutert die Art und Weise, wie die Negation in ihrem Reflexivwerden unter sich selbst fällt und sich so innerlich in ihren Gegensatz aus reflektierter Unmittelbarkeit (Schein) und selbstbezüglicher Negativität auseinanderlegt.

Zusammengefasst: Bezüglich ihrer Selbstbezüglichkeit ist die sich negierende Negation an sich selbst unmittelbar, weil mit sich selbst gleich. Bezüglich ihrer Selbstreferenzialität (ihrer Anwendung auf sich selbst) setzt die sich negierende Negation das Unmittelbare als Anderes zu ihr.

Daraus aber ergibt sich eine andere Formation des „Anderen“ bzw. der „Andersheit überhaupt“: „Das Andere ist hier also nicht das Seyn mit der Negati o n oder Grenze, sondern die Ne gation mit der Negation." (GW 11, S. 249) Als „internalisierte Andersheit" ${ }^{\text {109 }}$ macht die reine Form absoluter Reflexion die Grammatik des Anderen zu einem Effekt des Gleichen: Andersheit gegen die sich negierende Negation ist Produkt derselben und damit nichts anderes als diese, gerade wo aus ihr Andersheit hervorgeht. Das Nicht-Anders-Sein der Andersheit in der reinen absoluten Reflexion (analog zur Nicht-Unmittelbarkeit der Unmittelbarkeit im Wesen), wo die Unmittelbarkeit gegen die sich negierende Negation eine reflektierte ist, d.h. eine aufgehobene, markiert auch den Übergangspunkt, an dem Gleichheitsaspekt und Ungleichheitsaspekt der Reflexivität des Negativen, Selbstbezüglichkeit und Selbstreferenzialität, Unmittelbarkeit der reflexiven Negation und Unmittelbarkeit gegen die reflexive Negation in der Bewegung der einen ,absolute[n] Reflexion“ (GW 11, S. 250) zusammengehen und die Identität von „absoluter Reflexion“ und „absoluter Negativität“ bedeuten: Die Gleichheit der Negation, die diese schon an sich selbst hat, besteht gerade wesentlich darin, im Anderen gegen sie (der reflektierten Unmittelbarkeit) bei sich selbst anzukommen und so gerade in der Ungleichheit mit sich zusammenzugehen.

109 Iber: Metaphysik absoluter Relationalität, S. 125. 
Es ist jetzt entscheidend - deshalb ist diese Struktur hier auch so genau auseinandergelegt worden - zu sehen, in welcher Weise Hegel diese reine Form absoluter Reflexion weiter charakterisiert; eben hier fallen die berühmten Worte:

Das Werden im Wesen, seine reflectirende Bewegung, ist daher die Bewegung von Nichts zu Nichts, und dadurch zu sich selbst zurück. Das Uebergehen oder Werden hebt in seinem Uebergehen sich auf; das Andre, das in diesem Uebergehen wird, ist nicht das Nichtseyn eines Seyns, sondern das Nichts eines Nichts, und diß, die Negation eines Nichts zu seyn, macht das Seyn aus. - Das Seyn ist nur als die Bewegung des Nichts zu Nichts, so ist es das Wesen; und dieses hat nicht diese Bewegung in si ch, sondern ist sie als der absolute Schein selbst, die reine Negativität, die nichts ausser ihr hat, das sie negirte, sondern die nur ihr Negatives selbst negirt, das nur in diesem Negiren ist. (GW 11, S. 250)

Erneut ist auffällig, dass Hegel diese reine Form absoluter Reflexion mittels eines weiteren hinzugenommenen seinslogischen Terminus, den des „Nichts“ nämlich, zu beschreiben sucht. „Werden“, „Übergehen“, „Nichts“, „Anderes“, „Sein“ sind allesamt tragende Kategorien der Seinslogik, mit denen Hegel hier paradoxerweise eine Struktur beschreibt, die die „Erinnerung“ an die seinslogische Verantwortung, die der Schein etabliert hatte, an dieser Stelle vernachlässigt. Denn was Hegel hier beschreibt, ist gerade das Verschwinden von Andersheit in der absoluten Innerlichkeit des Wesens, in dessen reiner „absoluter Relationalität“ (Iber) ${ }^{110}$ bzw. „absoluter Kontextualität“" $(\mathrm{Gamm})^{111}$ nämlich alle Fixierungen bzw. gegeneinander festen Bestimmtheiten zu „Nichts“ werden. Alles löst sich hier in der einen reinen Bewegung bloßer Negativität auf. Hegel entwirft das Wesen als Prozessualität absoluter Bewegung, die gemäß der aristotelischen Bewegungsart der enérgeia, die ihr Ziel nicht außerhalb ihrer in einem Produkt hat, sondern in ihrem Vollzug selbst und so zugleich unendlich wie in jedem Punkt bereits vollendet ist, alle bestimmenden Gegensetzungen in der Einheit eines mit sich selbst erfüllten Vollzuges auslöscht. Dergestalt erscheint das Wesen, betrachtet als reine „absolute Reflexion“, als substanzloser reiner Selbstvollzug ohne vorausgehende Unmittelbarkeit, der das Bewegtsein akzidentell zukäme (,dieses [das Unmittelbare, C.W.] hat nicht die Bewegung in sich“). Zudem erscheint das Wesen in seiner „reine[n] Negativität“ zugleich als ,absolute[r] Schein“, weil diese gesamte Bewegung sich rein aus der reflexiven Selbstnegation des Negativen, d.h. rein aus sich selbst, erzeugt ${ }^{112}$ - so wie der Schein das Bestehen in und durch die eigene Negation ist. Wie radikal und konsequent Hegel dies denkt, zeigt sich daran, wie wichtig es ihm ist, nochmals zu betonen, dass die „reine Negativität [...] nichts ausser ihr hat, das sie negirte, sondern

110 Iber: Metaphysik absoluter Relationalität, S. 140.

111 Gamm: Der Deutsche Idealismus, S. 109.

112 Vgl. Iber: Metaphysik absoluter Relationalität, S. 130. 
die nur ihr Negatives selbst negirt, das nur in diesem Negiren ist“ (GW 11, S. 250). Die absolute Bewegung absoluter Vermittlung, die das Wesen ist, und die sich nur aus dem Funktionskern reiner selbstbezüglicher Negativität denken lässt, bleibt in den Momenten ihres Vollzuges ganz in sich, denn sie negiert sich einzig selbst: sodass die erste gegenständliche Negation, die etwas negiert, und die zweite reflexive Negation, welche wiederum die erste Negation negiert, an sich dasselbe sind, d.h. beide keinen anderen Gegenstand als sich selbst haben ${ }^{113}$ und deshalb den Gleichheits- bzw. Unmittelbarkeitsaspekt des Wesens verwirklichen. Zugleich geht erst aus der Beziehung der Selbstnegation und der Negation dessen, was sich aus der Selbstnegation ergibt (die reflektierte Unmittelbarkeit als Scheinen des Wesens in ihm selbst), die Sichselbstgleichheit des Negativen hervor: dass das negierte Negative „nur in diesem Negieren ist“, meint den Umstand, dass die Unmittelbarkeit der selbstbezüglichen Negation und des „Negativen überhaupt“ eben einzig darin zu finden ist, dass erst der Übergang der Negativität in ein Anderes (die Unmittelbarkeit) als Negationsbeziehung die Sich-selbst-Gleichheit der absoluten Negativität ermöglicht; sowohl das Negative der Negativität (die Unmittelbarkeit) als auch die Einheit der selbstbezüglichen Negativität, die sie nur im Bezug auf ihr Anderes findet, sind nur im emphatischen Sinn durch diese und in dieser Bewegung reiner Selbstnegation.

Für den minimalen kategorialen Realitätsbegriff ist das bisher eine äußerst unbefriedigende Bilanz: Die absolute Reflexion bleibt ganz und gar in sich, vollzieht sich widerstandslos an sich selbst und stößt damit in den Bewegungen ihrer reflexiven Selbstvermittlung an jedem Punkt wieder nur auf sich als Sich-selbst-Gleiches und kein Anderes. Wenn Hegel von dieser Stelle aus weiterkommen will, benötigt er einen Perspektivwechsel, der gewisse Implikationen dieser „logisch-autistischen“ Struktur so herausarbeitet, dass die seinslogische Verantwortlichkeit wieder aufgegriffen werden kann. Dies wird im Folgenden zu zeigen sein.

Der Begriff reiner, absoluter Reflexion führt also dazu, dass sich diese Reflexionsbewegung unmittelbar selbst aufhebt: dass also aufgrund der vollständigen Nichtigkeit von Andersheit überhaupt bezüglich ihres Andersseins und der vollständigen Auflösung aller fixierter Bestimmtheiten gegeneinander in dieser Bewegung kein wirkliches Übergehen zwischen Verschiedenen mehr stattfindet. Das Verschwinden der seinslogischen Verantwortlichkeit in der Bewegung absoluter Reflexion wird kontrastiv durch deren vollständige Formulierung in seinslogischen Termini nur umso deutlicher - und die Notwendigkeit, bei dieser subjektlosen, reinen, absolut prozessualen Form von Reflexion nicht stehen zu

113 Die reflexive Negation, welche die gegenständliche Negation negiert, ist zugleich die gegenständliche Negation, weil deren primärer Gegenstand einzig sie selbst ist. 
bleiben, wird dringlich markiert. Die Konturierung eines substanzlosen, absoluten und völlig selbstgenügsamen Formierungsprozesses reiner negativer Autogenerativität von Unterschieden aus Unterschieden, d.h. das Denken des absoluten Grundes absoluter Vermittlung, droht, in die grenzenlose, bestimmungslose Sichselbstgleichheit des Seins zurückzufallen, eben weil dieses Denken den begrenzten, begründeten und im Schein herausgearbeiteten Ansprüchen des Seins auf Andersheit nicht mehr genügen kann. Die nun folgende Entwicklung der Reflexionsformen als Stufenfolge, welche diese Kernstruktur absoluter Reflexion erneut gemäß der Pendelbewegung des Seins aufbricht, ohne bereits seinshafte, substanzielle Fixierungen in die Wesenslogik zurückzubringen, - wie es die Reflexionsbestimmungen später im Wesen in der „Erscheinung“ und der „Wirklichkeit“ in zunehmender Weise vollziehen -, erscheint aus dieser Perspektive als notwendige Konsequenz, um eine angemessene, entwicklungsfähige und problemorientierte kategoriale Logik von „Bestimmtheit überhaupt“ sowie weitergehend einen Begriff von minimaler kategorialer Realität zu entwerfen. Denn der reine minimale Begriff von „Realität überhaupt“ verlangt es, dass logisch Ungleichzeitiges synchronisiert und synthetisiert werden muss, ohne damit die generelle Entwicklungsrichtung der Logik (vom Sein über das Wesen zum Begriff) aufzuheben bzw. ohne konflikthaft eine zweite logische Ordnung der Kategorien zu etablieren: Seinslogische Andersheit muss als seinslogische Andersheit, d.h. mit einem widerständigen „Rest“ (vgl. Kap. 11 im Hauptteil II) an seinshafter Äußerlichkeit und Unmittelbarkeit, in den Beschreibungsrahmen des Wesens als reiner Reflexion integriert werden. Die Andersheit muss zu einem begrifflichen Element im Zusammenhang der Logik wesenslogischer absoluter Bestimmtheit werden, um einerseits die Vorgängigkeit des Realen überhaupt gegenüber dem (Subjekt-)Begrifflichen mit der andererseits ebenso vorhandenen Begriffsförmigkeit des vorgängigen Realen zusammenzudenken. Hegel hat die Möglichkeit einer solchen punktuellen Überlagerung der logischen Stadien zu Anfang der Wesenslogik von langer Hand vorbereitet, indem über die Stufe des Scheins seinslogische Andersheit und Unmittelbarkeit in einen unabweisbaren Verantwortlichkeitsanspruch transformiert worden sind, dem sich das reflexionslogische Denken zu stellen hat. Die Reflexionsformen werden diesen Verantwortlichkeitsanspruch auf der Höhe der Möglichkeiten wesenslogischen Denkens einlösen und in seiner vollständigen Anerkennung die partielle Asynchronizität des Logischen (seinslogische und wesenslogische Ansprüche) wieder vollständig synchronisieren. Damit wird die Homogenität des Wesens wieder so erreicht, dass von da an in der schrittweisen ganzheitlichen Zusammenführung von Wesen und Sein der Problemstand herausgearbeitet werden kann, der dann bezüglich der Perspektive eines vollständigen logischen Begriffs des Wirklichen den Übergang in die Logik des Begriffs notwendig macht. 